\title{
Energy dependence of the multiplicity moments at the LHC
}

\author{
G. R. Germano* and F. S. Navarra \\ Instituto de Física, Universidade de São Paulo, Rua do Matão, \\ 1371, CEP 05508-090, Cidade Universitária, São Paulo, SP, Brazil
}

(Received 3 November 2021; accepted 17 December 2021; published 5 January 2022)

\begin{abstract}
In this work, from the experimental data we evaluate the first $\mathrm{C}$-moments of the multiplicity distributions recently measured in proton-proton collisions at the LHC and compare them with the predictions of two models: the Kharzeev-Levin model and the Bialas-Praszalowicz model. We divide the data into three sets according to their phase space coverage: I: $p_{T}>100 \mathrm{MeV}$ and $|\eta|<0.5$; II: $p_{T}>100 \mathrm{MeV}$ and $|\eta|<2.4$ and III: $p_{T}>500 \mathrm{MeV}$ and $|\eta|<2.4$. The mean multiplicity grows with the energy according to a power law and the power is different for each set. The $C_{n}$ moments grow continuously with the energy, slowly in Set I and faster in the other sets. Except for KL in Set II, both models reproduce the main features of the data. The negative binomial parameter $k$ decreases continuously with the energy and there is no sign of change in this behavior.
\end{abstract}

DOI: 10.1103/PhysRevD.105.014005

\section{INTRODUCTION}

Particle production in hadronic collisions at very high energies is a very interesting phenomenon. On the experimental side particle multiplicities are easy to measure. On the theoretical side it is still a very challenging subject [1]. Most of the particles are produced with low and medium transverse momentum, where perturbative QCD cannot be applied and one has to use phenomenological models and/ or Monte Carlo event generators. In [2] it was shown that these generators are able to reproduce the main features of the multiplicity distributions but they are not yet able to describe the data with precision, especially in the large multiplicity region.

Over the past twenty years the QCD based theory of particle production has experienced a significant progress, especially because of the development of the color glass condensate (CGC) formalism [3]. One of the most interesting predictions of the CGC is that at very high energies the multiplicity distributions will become narrower [4]. This may be observed looking at the behavior of the multiplicity moments $C_{n}$, which should decrease with the energy. Ten years ago this prediction was confronted with the LHC data in [5], where a careful study of the moments was carried out. The conclusion was that the moments were continuously

\footnotetext{
*guilherme.germano@usp.br navarra@if.usp.br
}

Published by the American Physical Society under the terms of the Creative Commons Attribution 4.0 International license. Further distribution of this work must maintain attribution to the author(s) and the published article's title, journal citation, and DOI. Funded by SCOAP ${ }^{3}$. growing with the energy with no sign of change in this trend. Since then, new data appeared, taken at much higher energies, and it is time to check if there is anything special happening to the moments. Here it is important to mention that in the most recent experimental papers the multiplicity distributions $P(n)$ were presented but the moments $C_{n}$ were not. The first goal of this work is thus to compute the moments from the multiplicity tables, which we took from the hepdata.net databasis (the corresponding links are given right after the related articles in the reference list). The numerical values of these quantities are given in the tables contained in the Appendix. Then we will compare them with the predictions of two simple models. The first one, which we call Kharzeev-Levin (KL) was proposed in [6] and used in [7] to compute the moments of charged particle multiplicity distributions. This model is based on the BalitskyKovchegov (BK) equation with fixed dipole sizes. In the second model, called here Bialas-Praszalowicz (BP) model $[5,8]$, multiparticle production is described by a probability distribution, which is a superposition of a distribution of the number of sources and a Poisson distribution describing particle emission from each source. In the KL model there is always only one source, whereas in the BP model the number of sources grows with energy and can be large. In this aspect, these models are complementary.

The predictions of $\mathrm{KL}$ and $\mathrm{BP}$ models will be compared with the most recent data from the LHC on nonsingle diffractive pp collisions, which can be grouped into three sets:

(i) Set I: $p_{T}>100 \mathrm{MeV},|\eta|<0.5$, and energies $\sqrt{s}=900,2360$, and $7000 \mathrm{GeV}$, from CMS [2].

(ii) Set II: $p_{T}>100 \mathrm{MeV},|\eta|<2.4$, and energies $\sqrt{s}=900,7000$, and $8000 \mathrm{GeV}$ from ALICE [9]; 
$\sqrt{s}=8000 \mathrm{GeV} \quad[10]$ and $13000 \mathrm{GeV}$ [11] from ATLAS.

(iii) Set III: $p_{T}>500 \mathrm{MeV},|\eta|<2.4$, and energies $\sqrt{s}=900,7000 \mathrm{GeV}[12], 8000 \mathrm{GeV}(|\eta|<2.5)$ [10], $13000 \mathrm{GeV}(|\eta|<2.5)$ [13] from ATLAS and $13000 \mathrm{GeV}(|\eta|<2.4)[14]$ from CMS.

These datasets may contain particles produced through different production mechanisms. The particles measured in Set I are produced mainly from gluons; those measured in Set II come also from the fragmentation region (larger rapidities) and hence are produced also from the valence quarks.

Due to the larger transverse momentum cutoff, set III contains more particles which are produced perturbatively. These differences might lead to a different behavior of some observables. In previous analyses [2], it has been observed that multiplicity distributions in Set I satisfy the Koba-Nielsen-Olsen (KNO) scaling, whereas those in Set II do not.

\section{THE MODELS}

\section{A. The Kharzeev-Levin model}

In Refs. [6,7], the authors developed a model for multiplicity distributions based on the BK equation, which we will call KL model. They propose the following evolution equation for the parton multiplicity distribution $P_{n}$ :

$$
\frac{d P_{n}(Y)}{d Y}=-\Delta n P_{n}+(n-1) \Delta P_{n-1}(Y)
$$

which has the simple solution:

$$
P_{n}(Y)=P_{K L}(n)=e^{-\Delta Y}\left(1-e^{-\Delta Y}\right)^{n-1}
$$

where $Y=\ln (1 / x)$ and $\Delta$ is the BFKL Pomeron intercept, $\Delta=4 \ln 2 \bar{\alpha}_{s}$ with $\bar{\alpha}_{s}=\alpha_{s} N_{c} / \pi$. From the above expression we obtain the mean multiplicity:

$$
\langle n\rangle=\sum_{n} n P(n)=e^{\Delta Y}=\left(\frac{1}{x}\right)^{\Delta}
$$

The variable $x$ is defined here as in $[15,16]$ :

$$
x=\frac{q_{0}^{2}}{s}
$$

where $q_{0}$ is a constant. Inserting (4) into (3) we obtain:

$$
\langle n\rangle=\left(\frac{s}{q_{0}^{2}}\right)^{\Delta}
$$

The energy scale $q_{0}$ can be a mass or the average transverse momentum and hence it might be different for different datasets, but it should not depend on the collision energy $\sqrt{s}$. In what follows we will fix $q_{0}$ and $\Delta$ from the fit of the available experimental data.

\section{B. The Bialas-Praszalowicz model}

In the BP model the multiplicity distribution is given by:

$$
P_{B P}(n)=\int_{0}^{\infty} d t F(t) e^{-\bar{n} t} \frac{(\bar{n} t)^{n}}{n !} .
$$

where $t$ is a fraction of the average multiplicity, and $F(t)$ the distribution of sources that contribute a fraction $t$ to the multiplicity probability $P_{B P}(n)$. The function $F$ is normalized:

$$
\int_{0}^{\infty} d t F(t)=\int_{0}^{\infty} d t t F(t)=1 .
$$

The above equation implies that $\langle n\rangle=\bar{n}$. In the BP model $F$ is given by

$$
F(t, k)=\frac{k^{k}}{\Gamma(k)} t^{k-1} e^{-k t}
$$

where $\Gamma$ is the gamma function and $P_{B P}$ turns out to be the negative binomial distribution (NBD) with parameter $k$, which is known to describe relatively well the data at lower energies [17]. From the equations above we see that in the BP model particles are emitted by several sources, whose number follows the distribution given by the function $F$ and increases with the energy. Moreover, each source emits particles according to a Poisson distribution.

Since the late 1980s it has been known that a single negative binomial distribution cannot fully describe the measured multiplicity distributions. Indeed, up to $\sqrt{s}=1 \mathrm{TeV}, P(n)$ was reasonably well described by a NBD. At the Tevatron at $\sqrt{s}=1.8 \mathrm{TeV}$, the appearance of a shoulder in $P(n)$ was confirmed and this led to the twocomponent model proposed in [18] in which the measured data were described by a combination of two NBDs, one representing the soft and one the semihard component. The success of the double NBD approach was later confirmed by the $\sqrt{s}=7 \mathrm{TeV}$ LHC data [19]. More recently, the data taken at $\sqrt{s}=8$ and $13 \mathrm{TeV}$ have shown that the best fit is obtained with the inclusion of a third negative binomial distribution [20-22]. Curiously, in [5] a good fit of the measured $C_{n}$ moments was obtained with the single negative binomial (SNB) approach. One of our goals in this work is to check at which energy and in which part of the phase space the SNB fails to reproduce the most recent data.

Distribution (8) depends on one parameter $k$, which depends on the collision energy. The analysis of lower energy data shows that $k$ decreases with increasing energy. 

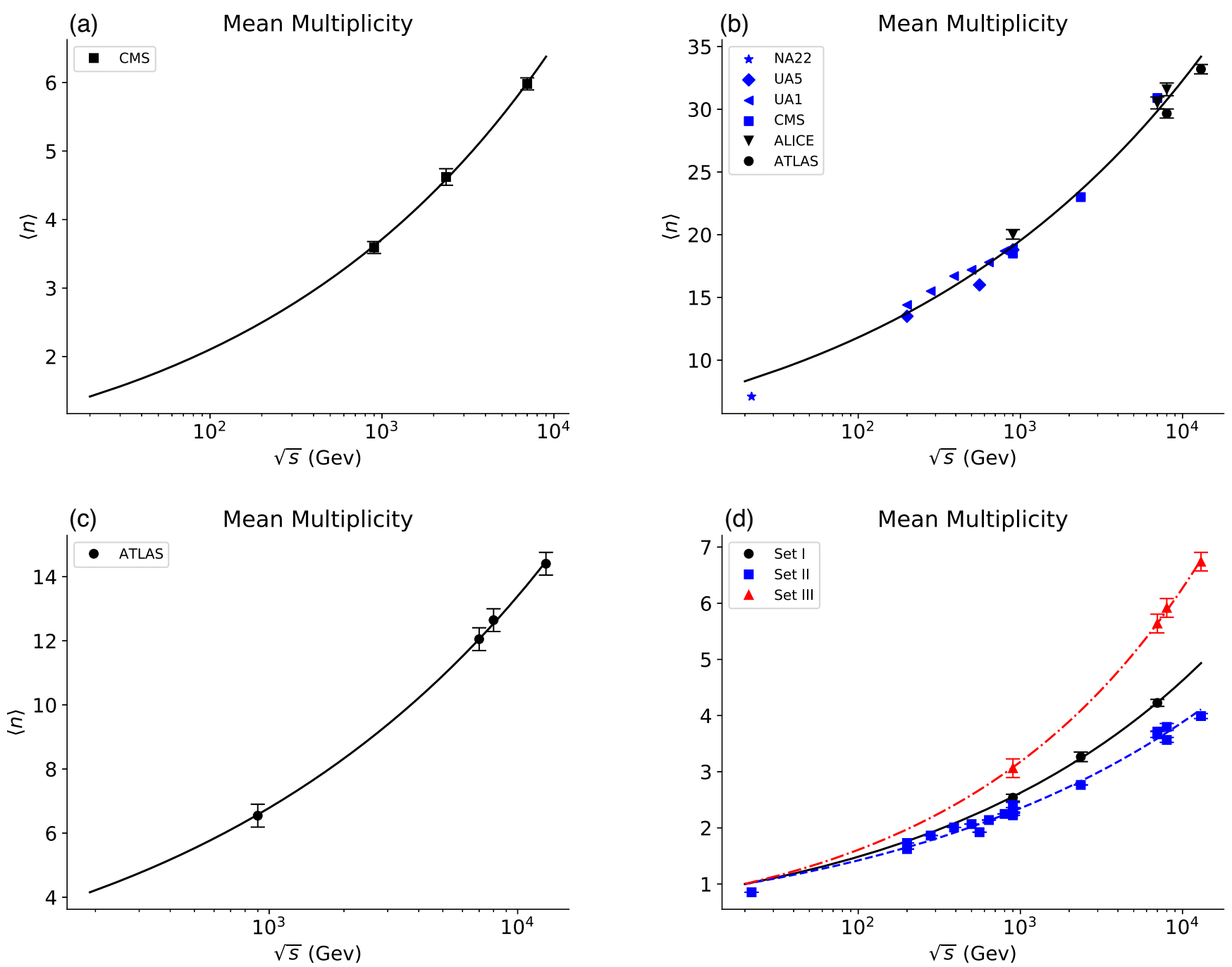

FIG. 1. Mean multiplicities fitted with (5). (a) Dataset I, taken from Ref. [2]. (b) Dataset II, taken from Ref. [10,11] (circles), from Ref. [9] (down-triangles), from Ref. [2] (squares), from Ref. [23] (left-triangles), from Ref. [24] (diamonds) and from Ref. [25] (stars). (c) Dataset III, taken from Ref. [10,12,13] (circles). (d) Mean multiplicities adjusted with (5) for all Sets. The points in each set were multiplied by a constant to illustrate the different behavior with the energy.

When $k=1$ the probability distribution $P_{\mathrm{NBD}}$ becomes a geometrical distribution $P(n)=\langle n\rangle^{n} /(1+\langle n\rangle)^{n+1}$ When $k$ is large $(1 / k \rightarrow 0)$, the distribution $P_{\mathrm{NBD}}$ tends to a Poisson distribution.

\section{RESULTS}

\section{A. The mean multiplicity}

We start fitting the data on the mean multiplicity as a function of the energy with the help of the parametrization (5), as shown in Fig. 1. For completeness we also show data taken at lower energies. The obtained values of $q_{0}$ and $\Delta$ are presented in Table I. As it can be seen in the figure, the data are well reproduced by the power law (5). However, there is a difference in the size of the parameters. For Set II, the power $\Delta$ is smaller and the corresponding energy dependence is weaker than in the other sets. This seems to be a feature of the data. To illustrate this, in Fig. 1(d) we have have multiplied the data and the fitting curves by appropriate constants so that the curves start at the same point. The resulting plot shows clearly the different energy behavior of the three sets. Since in Sets II and III the rapidity coverage is the same, the difference in the energy behavior of the mean multiplicity must be related to the difference in the lower $p_{T}$ cut. As noted in the introduction, the most natural explanation (if not the only one) for this

TABLE I. Parameters $q_{0}$ and $\Delta$ used in Eq. (5).

\begin{tabular}{lcc}
\hline \hline Set & $\Delta$ & $q_{0}(\mathrm{GeV})$ \\
\hline I & 0.13 & 6.31 \\
II & 0.11 & 0.01 \\
III & 0.16 & 4.83 \\
\hline \hline
\end{tabular}


energy behavior is the increase of the contribution of perturbative events, which are known to have a strong energy dependence. It is nevertheless remarkable how a relatively modest increase in $p_{T}$ (which is here still far from the typical few $\mathrm{GeV}$ region) can produce a visible effect.

The data of Sets I and II have the same lower $p_{T}$ cut and very different rapidity coverage. In Set I, we observe particles produced in the central (rapidity) region, which is dominated by gluons. In Set II there is a larger contribution coming from the fragmentation region, where the valence quarks play an important role in particle production. The separation of central and fragmentation regions and its relevance for particle production was first discussed in [26] and later implemented as a model in [27]. In the fragmentation region, partons from the projectile and from the target collide in a very asymmetric kinematical configuration. If a parton from the projetile carries a large momentum fraction of the proton, the one from the target carries a very small fraction of the target proton momentum. Therefore, data in this region of the phase space are more sensitive to the low-x QCD dynamics and to saturation effects. As it is well known, saturation tames the growth of observables (parton distribution functions, color dipole scattering amplitudes, hadron cross sections, etc...) with the energy. According to these ideas, it is tempting to interpret the weaker energy dependence of the data of Set II (as compared to Set I) as a manifestation of low x saturation effects.

\section{B. The $C_{n}$ moments}

The moments are defined as:

$$
C_{m}=\frac{\left\langle n^{m}\right\rangle}{\langle n\rangle^{m}}
$$

They have been measured at the LHC and explicitly given in Refs. [2,28]. For higher energies we can calculate them from the hepdata.net databasis, as we did in the case of the mean multiplicities. In the KL model we can obtain the moments from the definition (9) and the multiplicity distribution (2). The first of them are given by:

$$
\begin{aligned}
& C_{2}=2-\frac{1}{\langle n\rangle} \\
& C_{3}=\frac{6(\langle n\rangle-1)\langle n\rangle+1}{\langle n\rangle^{2}}, \\
& C_{4}=\frac{(12\langle n\rangle(\langle n\rangle-1)+1)(2\langle n\rangle-1)}{\langle n\rangle^{3}} \\
& C_{5}=\frac{(\langle n\rangle-1)\left(120\langle n\rangle^{2}(\langle n\rangle-1)+30\langle n\rangle\right)+1}{\langle n\rangle^{4}}
\end{aligned}
$$

Since the mean multiplicities are given by (5), there are no free parameters in the calculation with the KL model. In the
TABLE II. Parameters $a$ and $b$ obtained from the fit of data with (13), for the three sets.

\begin{tabular}{lcc}
\hline \hline Set & $a$ & $b$ \\
\hline I & 1.68 & 0.02 \\
II & 0.97 & 0.08 \\
III & 1.30 & 0.06 \\
\hline \hline
\end{tabular}

BP model the moments are obtained from (6) and the first moments $C_{n}$ are given by:

$$
\begin{gathered}
C_{2}=\frac{1}{\langle n\rangle}+1+\frac{1}{k} \rightarrow \frac{1}{k}=C_{2}-1-\frac{1}{\langle n\rangle} . \\
C_{3}=C_{2}\left(2 C_{2}-1\right)-\frac{C_{2}-1}{\langle n\rangle}, \\
C_{4}=C_{2}\left(6 C_{2}^{2}-7 C_{2}+2\right)-2 \frac{3 C_{2}^{2}-4 C_{2}+1}{\langle n\rangle}+\frac{C_{2}-1}{\langle n\rangle^{2}}, \\
C_{5}=C_{2}\left(24 C_{2}^{3}-46 C_{2}^{2}+29 C_{2}-6\right) \\
-2 \frac{18 C_{2}^{3}-34 C_{2}^{2}+19 C_{2}-3}{\langle n\rangle} \\
+\frac{14 C_{2}^{2}-23 C_{2}+9}{\langle n\rangle^{2}}-\frac{C_{2}-1}{\langle n\rangle^{3}} .
\end{gathered}
$$

In the above expressions, we need to know $k$ to compute the moment $C_{2}$ and then all the other moments. Instead of choosing values for $k$, we follow [5] and parametrize $C_{2}$ as

$$
C_{2}=a+b \log (\sqrt{s}[\mathrm{GeV}])
$$

We carefully repeat the fitting procedure described in Ref. [5]. We insert Eq. (13) into the second line of Eq. (12) obtaining $C_{4}$ as a function of $a$ and $b$. Then, $C_{4}$ is adjusted to the data and $a$ and $b$ are determined. After that, $C_{2}, C_{3}$ and $C_{5}$ are calculated and compared to the data. At last, inserting $C_{2}$ and $\langle n\rangle$ [given by Eq. (5)] into Eq. (11), we find $k$. The obtained values are listed in Table II. Having determined the parameters $a$ and $b$, which are energy independent, we can calculate all the first $\mathrm{C}$ moments, compare them with data and make predictions. This is shown in Fig. 2, where we compare the KL and BP moments with the three datasets. Looking first at the data (which are put together here for the first time) we observe that in all figures the moments grow with the energy. The moments from Set I grow much slower and are even compatible with a constant value. This motivated the observation made in Ref. [2], where the authors claimed that data relative to small rapidities exhibit KNO scaling $[1,29]$, while data of larger rapidity intervals do not. Comparing the moments obtained with Sets II and III we see that the $C_{n}$ 's grow with energy in the same (strong) way. 
Both models give a reasonable description of data. The $\mathrm{KL}$ model has less freedom than the $\mathrm{BP}$ one, since in the latter, apart from $\langle n\rangle$, we can also adjust $C_{2}$ to the data. This flexibility yields better fits. In [5] the mean multiplicity was calculated from the central rapidity density, $d N / d \eta(\eta=0)$, while here we have used (5). We believe that this procedure is more accurate. The results support the BP picture, in which multiple sources (the number of sources follows the distribution $F$ ) produce a number of particles which follows a Poisson distribution and both distributions depend on the energy. On the other hand the success of the KL model would imply that particle production comes mostly from gluon cascading resulting ultimately from the BK equation. In view of its simplicity, the KL model does a reasonable job with Sets I and III and fails badly with Set II. In this set we have the bigger multiplicities and, as already pointed out in [7], with the expressions (10) we rapidly reach the asymptotic values of the "large $\langle n\rangle$ " limit. The resulting moments are large and very flat functions of $\sqrt{s}$, in contradiction with the data. It is not clear which part of the model is wrong or incomplete. Having in mind the observations made in the case of the mean multiplicities, we would guess that there is a missing component in this version of the model, namely the contribution from the fragmentation region. Increasing the lower $p_{T}$ cut and selecting higher $p_{T}$ particles, we reduce the contribution of the fragmentation region yield (which is mostly forward and with low transverse momentum) and improve the agreement between the KL model and data, as is indeed seen in the comparison with Set III data. Finally, it is worth mentioning that the discrepancy between the KL model and the Set II data is visible not only in the $C_{n}$ moments but also in the entire distribution $P(n)$, as shown in [30].

Finally, having fitted $\langle n\rangle$ and $C_{2}$ we return to (11) and plot $1 / k$ as a function of $\sqrt{s}$. The result, shown in Fig. 3 indicates that $1 / k$ is an increasing function and there is no sign of a different behavior. These findings extend the conclusions found ten years ago in [5] to the present energies, which are two times higher.

\section{CONCLUSIONS}

We have used the KL (10) and BP models (12) to fit the multiplicity moments measured recently at the LHC. The moments in the KL model depend only on $\langle n\rangle$ and hence only on two free parameter $q_{0}$ and $\Delta$. Even so it reproduces reasonably well the moments of the data of Sets I and III (where the model is indeed expected to be valid as we have mainly gluons in the perturbative regime). The failure in describing the data of Set II is arguably due to the absence of a component from the fragmentation region.

In the BP model (12) and assuming a logarithmic growth (13) of $C_{2}$ moment, we have been able to reproduce the multiplicity moments over the wide range of energies for different rapidity intervals. The input growth of $C_{2}$ with energy can be translated through (11) into a decrease of the parameter $k$. This behavior is consistent with lower energies and does not exhibit the change predicted in [4].

\section{ACKNOWLEDGMENTS}

We are grateful to the Brazilian funding agencies $\mathrm{CNPq}$ and CAPES and also to the INCT-FNA.

\section{APPENDIX: MULTIPLICITIES AND C-MOMENTS}

We present below the numerical values of the $\langle n\rangle$ 's and $C_{n}$ 's, which appear in the figures (Tables III-V). Some of them (for the LHC energies) we have calculated from the data, as described in the text.

TABLE III. $\langle n\rangle$ and $C_{n}$ for Set I.

\begin{tabular}{lccc}
\hline \hline$\sqrt{s}(\mathrm{GeV})$ & 900 & 2360 & 7000 \\
\hline$\langle n\rangle$ & $3.59 \pm 0.09$ & $4.62 \pm 0.12$ & $5.98 \pm 0.09$ \\
$\mathrm{C} 2$ & $1.98 \pm 0.11$ & $1.96 \pm 0.12$ & $2.04 \pm 0.07$ \\
$\mathrm{C} 3$ & $5.41 \pm 0.43$ & $5.35 \pm 0.46$ & $5.86 \pm 0.28$ \\
$\mathrm{C} 4$ & $18.58 \pm 1.96$ & $18.38 \pm 2.11$ & $21.15 \pm 1.36$ \\
$\mathrm{C} 5$ & $75.81 \pm 10.03$ & $74.50 \pm 10.82$ & $90.14 \pm 7.21$ \\
\hline \hline
\end{tabular}



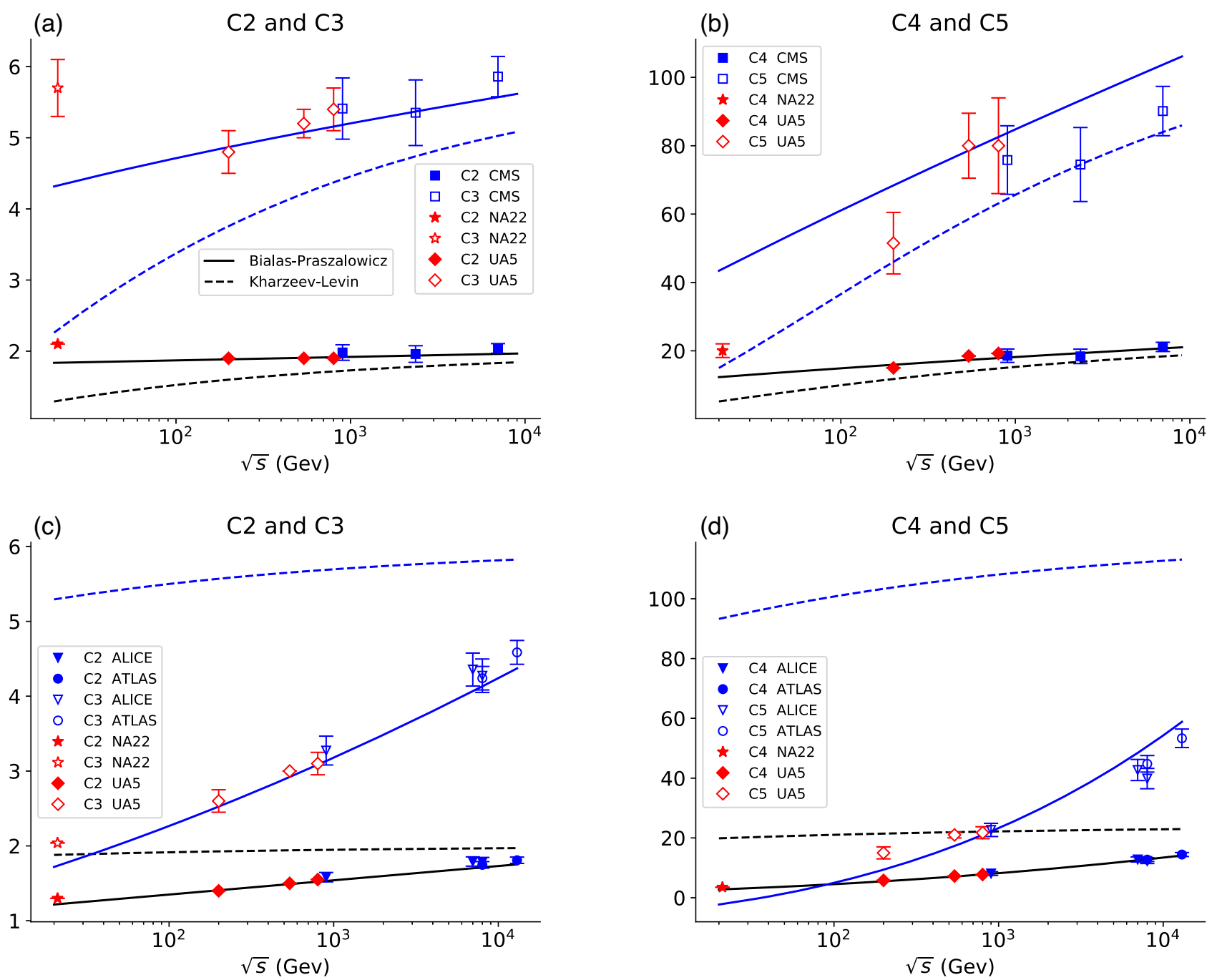

(d)

\section{C4 and C5}

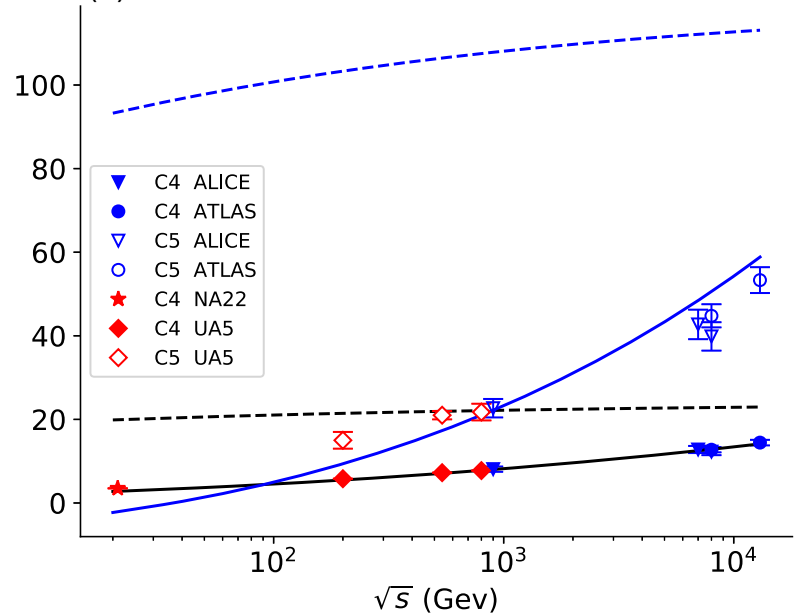

(e)

$\mathrm{C} 2$ and $\mathrm{C} 3$
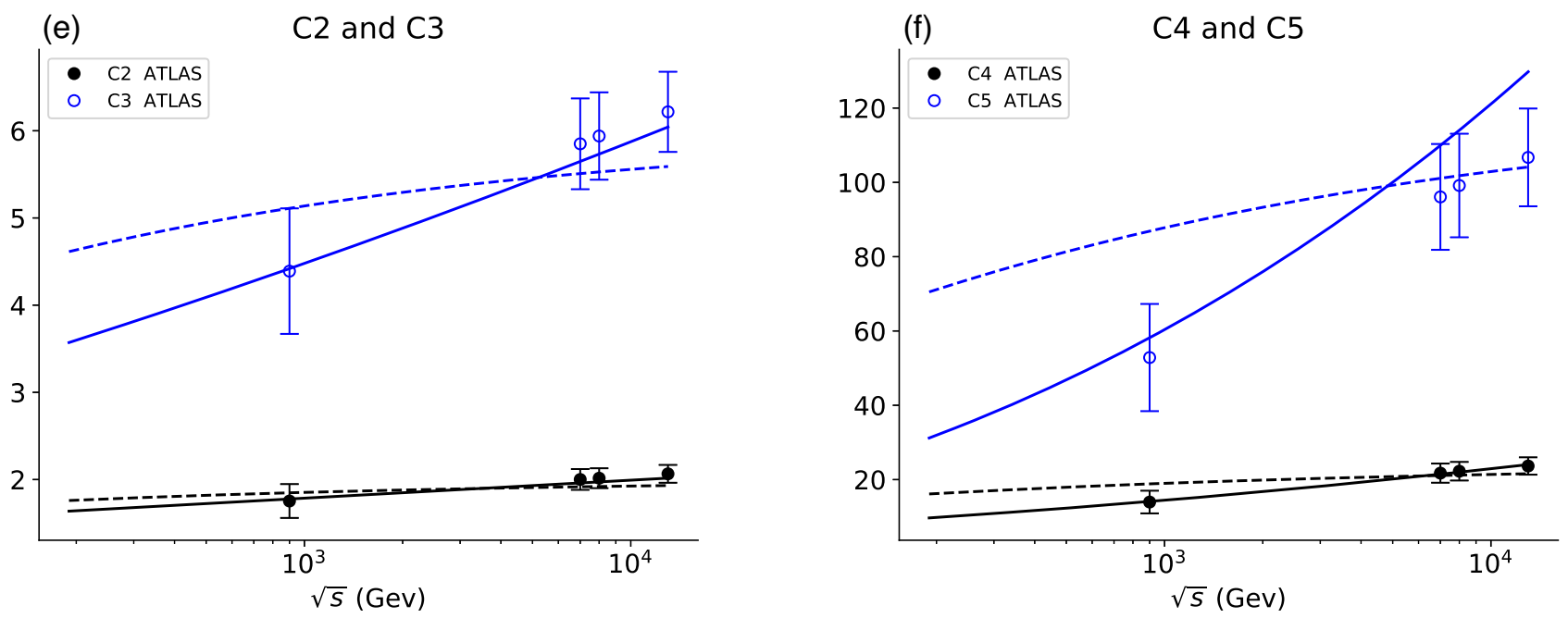

FIG. 2. $\quad C_{n}$ moments. Solid lines: BP model. Dashed lines: KL model. (a) $C_{2}$ and $C_{3}$. Set I. Data from [25] (stars), [24] (diamonds) and [2] (squares). (b) $C_{4}$ and $C_{5}$. Set I. Data from [25] (stars), [24] (diamonds), and [2] (squares). (c) $C_{2}$ and $C_{3}$. Set II. Data from [25] (stars), [24] (diamonds), [9] (triangles) and [10,11] (circles). (d) $C_{4}$ and $C_{5}$. Set II. Data from [25] (stars), [24] (diamonds), [9] (triangles) and [10,11] (circles). (e) $C_{2}$ and $C_{3}$. Set III. Data from [10,12,13]. (f) $C_{4}$ and $C_{5}$. Set III. Data from $[10,12,13]$. 


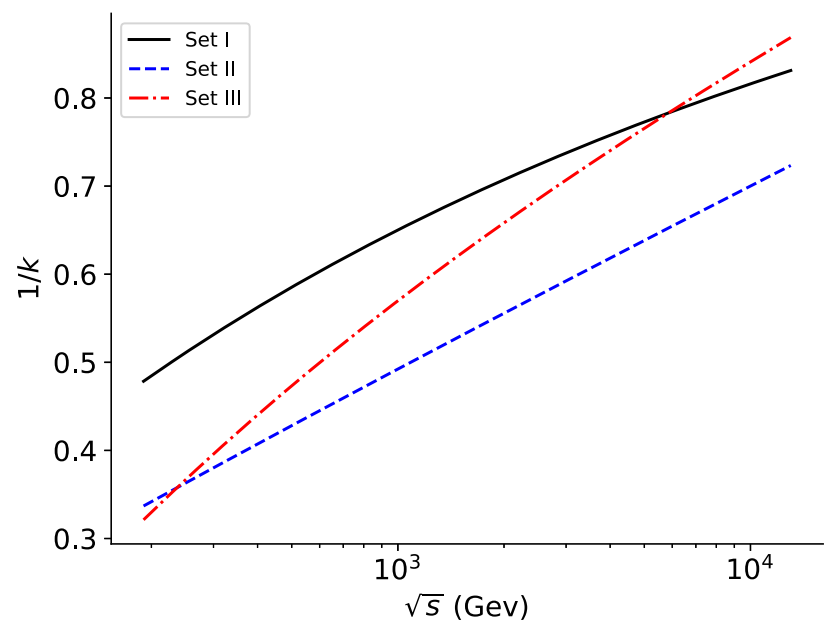

FIG. 3. $1 / k$ calculated with $(11)$ as a function of the collision energy for the three different sets.

TABLE IV. $\langle n\rangle$ and $C_{n}$ for Set II.

\begin{tabular}{lccccc}
\hline \hline$\sqrt{s}(\mathrm{GeV})$ & 900 & 7000 & 8000 (ALICE) & 8000 (ATLAS) & 13000 \\
\hline$\langle n\rangle$ & $20.03 \pm 0.38$ & $30.51 \pm 0.48$ & $31.59 \pm 0.51$ & $29.66 \pm 0.36$ & $33.20 \pm 0.37$ \\
$\mathrm{C} 2$ & $1.58 \pm 0.06$ & $1.79 \pm 0.06$ & $1.78 \pm 0.06$ & $1.75 \pm 0.04$ & $1.81 \pm 0.04$ \\
$\mathrm{C} 3$ & $3.27 \pm 0.19$ & $4.36 \pm 0.22$ & $4.27 \pm 0.22$ & $4.24 \pm 0.16$ & $4.59 \pm 0.16$ \\
$\mathrm{C} 4$ & $8.10 \pm 0.63$ & $12.80 \pm 0.85$ & $12.28 \pm 0.84$ & $12.75 \pm 0.63$ & $14.44 \pm 0.67$ \\
$\mathrm{C} 5$ & $22.68 \pm 2.21$ & $42.72 \pm 3.53$ & $39.85 \pm 3.40$ & $44.78 \pm 2.77$ & $53.32 \pm 3.09$ \\
\hline \hline
\end{tabular}

TABLE V. $\langle n\rangle$ and $C_{n}$ for Set III.

\begin{tabular}{lcccc}
\hline \hline$\sqrt{s}(\mathrm{GeV})$ & 900 & 7000 & 8000 & 13000 \\
\hline$\langle n\rangle$ & $6.54 \pm 0.36$ & $12.05 \pm 0.36$ & $12.65 \pm 0.36$ & $14.41 \pm 0.35$ \\
$\mathrm{C} 2$ & $1.75 \pm 0.19$ & $2.00 \pm 0.12$ & $2.02 \pm 0.11$ & $2.07 \pm 0.10$ \\
$\mathrm{C} 3$ & $4.39 \pm 0.72$ & $5.85 \pm 0.52$ & $5.94 \pm 0.50$ & $6.22 \pm 0.46$ \\
$\mathrm{C} 4$ & $13.95 \pm 3.05$ & $21.74 \pm 2.58$ & $22.25 \pm 2.50$ & $23.66 \pm 2.33$ \\
$\mathrm{C} 5$ & $52.85 \pm 14.43$ & $96.08 \pm 14.25$ & $99.15 \pm 13.94$ & $106.72 \pm 13.15$ \\
\hline \hline
\end{tabular}

[1] J. F. Grosse-Oetringhaus and K. Reygers, J. Phys. G 37, 083001 (2010).

[2] V. Khachatryan et al. (CMS Collaboration), J. High Energy Phys. 01 (2011) 079.

[3] F. Gelis, E. Iancu, J. Jalilian-Marian, and R. Venugopalan, Annu. Rev. Nucl. Part. Sci. 60, 463 (2010).

[4] F. Gelis, T. Lappi, and L. McLerran, Nucl. Phys. A828, 149 (2009).

[5] M. Praszalowicz, Phys. Lett. B 704, 566 (2011).

[6] E. Levin and M. Lublinsky, Nucl. Phys. A730, 191 (2004).

[7] D. E. Kharzeev and E. M. Levin, Phys. Rev. D 95, 114008 (2017).

[8] A. Bialas, Acta Phys. Pol. B 41, 2163 (2010).
[9] S. Acharya et al. (ALICE Collaboration), Eur. Phys. J. C 77, 852 (2017).

[10] G. Aad et al. (ATLAS Collaboration), Eur. Phys. J. C 76, 403 (2016).

[11] G. Aad et al. (ATLAS Collaboration), Eur. Phys. J. C 76, 502 (2016).

[12] G. Aad et al. (ATLAS Collaboration), New J. Phys. 13, 053033 (2011).

[13] G. Aad et al. (ATLAS Collaboration), Phys. Lett. B 758, 67 (2016).

[14] A. M. Sirunyan et al. (CMS Collaboration), Eur. Phys. J. C 78, 697 (2018).

[15] J. Bartels, E. Gotsman, E. Levin, M. Lublinsky, and U. Maor, Phys. Lett. B 556, 114 (2003). 
[16] F. Carvalho, F. O. Duraes, V. P. Goncalves, and F. S. Navarra, Mod. Phys. Lett. A 23, 2847 (2008).

[17] For a review see: W. Kittel and E. A. De Wolf, Soft Multihadron Dynamics (World Scientific, Singapore, 2005).

[18] A. Giovannini and R. Ugoccioni, Phys. Rev. D 59, 094020 (1999); 69, 059903(E) (2004); 68, 034009 (2003).

[19] P. Ghosh, Phys. Rev. D 85, 054017 (2012).

[20] I. Zborovsky, Eur. Phys. J. C 78, 816 (2018); J. Phys. G 40, 055005 (2013).

[21] M. Biyajima and T. Mizoguchi, Int. J. Mod. Phys. A 34, 1950203 (2019).

[22] M. Rybczynski, G. Wilk, and Z. Włodarczyk, Phys. Rev. D 99, 094045 (2019).
[23] C. Albajar et al. (UA1 Collaboration), Nucl. Phys. B335, 261 (1990).

[24] R. E. Ansorge et al. (UA5 Collaboration), Phys. Lett. 160B, 193 (1985); Z. Phys. C 43, 357 (1989).

[25] M. Adamus et al. (EHS/NA22 Collaboration), Z. Phys. C 37, 215 (1988).

[26] L. Van Hove and S. Pokorski, Nucl. Phys. B86, 243 (1975).

[27] G. N. Fowler, F. S. Navarra, M. Plumer, A. Vourdas, R. M. Weiner, and G. Wilk, Phys. Rev. C 40, 1219 (1989).

[28] K. Aamodt et al. (ALICE Collaboration), Eur. Phys. J. C 68, 89 (2010).

[29] Z. Koba, H. B. Nielsen, and P. Olesen, Nucl. Phys. B40, 317 (1972).

[30] G. R. Germano and F. S. Navarra, arXiv:2011.08912. 